\title{
E. coli chaperones DnaK, Hsp33 and Spy inhibit bacterial functional amyloid assembly
}

\author{
Margery L. Evans, ${ }^{1}$ Jens C. Schmidt, ${ }^{1}$ Marianne Ilbert,, Shannon M. Doyle, ${ }^{2}$ Shu Quan,, James C.A. Bardwell,, Ursula Jakob,
} Sue Wickner ${ }^{2}$ and Matthew R. Chapman ${ }^{1, *}$

\begin{abstract}
'Department of Molecular, Cellular and Developmental Biology; University of Michigan; Ann Arbor, MI USA; ${ }^{2}$ Laboratory of Molecular Biology; National Institutes of Health; Bethesda, MD USA
\end{abstract}

Key words: chaperone, curli, functional amyloid, CsgA, DnaK, Hsp33, Spy

\begin{abstract}
Amyloid formation is an ordered aggregation process, where $\beta$-sheet rich polymers are assembled from unstructured or partially folded monomers. We examined how two Escherichia coli cytosolic chaperones, DnaK and Hsp33, and a more recently characterized periplasmic chaperone, Spy, modulate the aggregation of a functional amyloid protein, CsgA. We found that DnaK, the Hsp70 homolog in E. coli, and Hsp33, a redox-regulated holdase, potently inhibited CsgA amyloidogenesis. The Hsp33 anti-amyloidogenesis activity was oxidation dependent, as oxidized Hsp33 was significantly more efficient than reduced Hsp33 at preventing CsgA aggregation. When soluble CsgA was seeded with preformed amyloid fibers, neither Hsp33 nor DnaK were able to efficiently prevent soluble CsgA from adopting the amyloid conformation. Moreover, both DnaK and Hsp33 increased the time that CsgA was reactive with the amyloid oligomer conformation-specific A11 antibody. Since CsgA must also pass through the periplasm during secretion, we assessed the ability of the periplasmic chaperone Spy to inhibit CsgA polymerization. Like DnaK and Hsp33, Spy also inhibited CsgA polymerization in vitro. Overexpression of Spy resulted in increased chaperone activity in periplasmic extracts and in reduced curli biogenesis in vivo. We propose that DnaK, Hsp33 and Spy exert their effects during the nucleation stages of CsgA fibrillation. Thus, both housekeeping and stress induced cytosolic and periplasmic chaperones may be involved in discouraging premature CsgA interactions during curli biogenesis.
\end{abstract}

\section{Introduction}

Amyloid formation is central to human ailments such as Alzheimer disease, Parkinson disease, Huntington disease, transmissible spongiform encephalopathies and type II diabetes., ${ }^{1,2}$ Proteins and peptides of various primary sequence signatures can assemble into amyloids, which are biophysically distinguished as 4-12 $\mathrm{nm}$ wide, $\beta$-sheet rich fibers that are highly resistant to denaturation and bind to dyes such as thioflavin T (ThT) and Congo red (CR) ${ }^{3,4}$ Amyloid subunits spontaneously polymerize into amyloid fibers in vitro after a defined lag phase, which is followed by rapid fiber growth. ${ }^{5-7}$ Although many proteins, if not all, can adopt the amyloid conformation in vitro, certain amino acid sequences or cellular environments significantly influence in vivo amyloidogenesis. $1,6,8$

A new class of "functional" amyloids has been linked to important physiological processes in cells. Unlike disease-associated amyloid formation in vivo, which is sporadic and difficult to predict, the formation of "functional" amyloids is a tightly regulated cellular process. ${ }^{9} 10$ Functional amyloids have been identified in nearly all walks of life, from bacteria ${ }^{11-14}$ to fungi ${ }^{15-17}$ to mammals..$^{18,19}$ Understanding how functional amyloids can form without apparent cellular toxicity will provide new insights into the processes that break down during disease-associated amyloid formation.
The first described functional amyloid was curli, extracellular organelles assembled by enteric bacteria such as Escherichia coli and Salmonella species. ${ }^{12}$ Curli fibers play an important roll in biofilm formation, ${ }^{20-22}$ cell adhesion, ${ }^{23}$ cell invasion ${ }^{24,25}$ and induction of the host inflammatory response. ${ }^{26-28}$ Curli biogenesis is a highly regulated process and requires the products of two divergently transcribed operons $(\operatorname{csg} B A C$ and $\operatorname{csg} D E F G) .{ }^{29}$ $\mathrm{CsgD}$ is a transcriptional activator of the $\operatorname{csg} B A C$ operon. ${ }^{29,30}$ $\mathrm{CsgG}$ is an outermembrane lipoprotein required for the secretion of the major and minor curli subunits CsgA and CsgB, respectively. ${ }^{30,31} \mathrm{Csg} \mathrm{A}$ is secreted as a soluble, unstructured peptide and is nucleated into an extracellular amyloid fiber by the cell surface-associated CsgB..$^{12,32-34} \mathrm{CsgF}$ assists in attachment of CsgA fibers to the cell surface and aids $\mathrm{CsgB}$ in its nucleation function. ${ }^{35} \mathrm{CsgE}$ functions as a specificity factor in the periplasm for CsgG-mediated secretion of curli subunits and possesses chaperone activity in vitro. ${ }^{36} \mathrm{CsgC}$ putatively collaborates with $\mathrm{Csg} G$ to regulate the passage of proteins and small molecules through CsgG. ${ }^{37,38}$ Curli biogenesis must be a finely tuned system in order to prevent intracellular aggregation of CsgA.

The primary amino acid sequence of CsgA features five imperfect repeating units that are predicted to form a $\beta$-helix with an overall cross $\beta$-strand structure. ${ }^{39,40}$ Repeating units one and five are highly amyloidogenic and contain conserved glutamine and 
asparagine residues that promote amyloid formation..$^{41}$ Repeating units two, three and four contain gatekeeper residues, which reduce the propensity to form amyloids on their own. ${ }^{42}$ The separation of the amyloidogenic regions of CsgA is believed to help reduce the occurrence of premature amyloid formation within the cell. CsgA also contains a Sec-secretion signal sequence and an $\mathrm{N}$-terminal 22 amino acid sequence on the mature protein that is required for CsgG-mediated translocation across the outermembrane. ${ }^{31,36}$ Both CsgA and CsgB proteins are at undetectable levels by protein gel blot in the absence of $\mathrm{CsgG}^{30}{ }^{30}$ Whether this is due to proteolytic degradation of CsgA and CsgB or transcriptional repression remains to be established.

During synthesis and export, CsgA may be exposed to the cytosolic and periplasmic chaperone machinery, which could modulate amyloidogenesis by preventing inappropriate amyloid fiber formation within the cell. How the cellular chaperone machinery engages amyloidogenic proteins, especially functional amyloids, is poorly understood. Recent studies have shown that the human chaperone Hsp70 partially prevents amyloid formation of the disease-associated proteins $A \beta(1-42)$ and $\alpha$-synuclein. ${ }^{43,44} \mathrm{Hsp} 70$ is an ATPase but can inhibit A $\beta$ amyloidogenesis in the absence of ATP and its cochaperone Hsp $40 .{ }^{45}$ DnaK, the Hsp70 homolog in E. coli, ${ }^{46}$ has been shown to prevent protein aggregation and assist in the refolding of denatured proteins or remodeling of large protein aggregates. ${ }^{47,48}$ DnaK is composed of two functional domains, the ATPase domain and the substrate-binding domain. ${ }^{49}$ Substrate binding and release by DnaK is regulated by the nature of the nucleotide bound to the ATPase domain..$^{50,51}$ The ATP bound form of DnaK has a significantly lower substrate affinity than the ADP bound form. ${ }^{49}$ The ATPase cycle of DnaK is regulated by the co-chaperones DnaJ and GrpE. ${ }^{47}$ DnaJ stimulates the DnaK ATPase activity, while GrpE is a nucleotide exchange factor that stimulates the release of ADP from DnaK..$^{52,53}$ DnaJ, or a DnaJ homolog, and GrpE are essential for DnaK activity in vivo and in in vitro luciferase disaggregation assays. ${ }^{54}$

Hsp33 is a "holdase" chaperone..$^{55}$ Holdase chaperones are not thought to actively refold denatured substrates, but instead quench exposed hydrophobic regions of proteins during periods of transient cellular stress. ${ }^{55} \mathrm{Hsp} 33$ expression is controlled by $\sigma^{32}$, the heat shock transcription initiation factor, and is induced at elevated temperatures and other conditions that trigger protein denaturation. ${ }^{56} \mathrm{Hsp} 33$ is activated at the protein level by oxidizing and denaturing conditions. ${ }^{55}$ Four cysteines located in Hsp33's C-terminal redox-switch domain coordinate $\mathrm{Zn}^{2+}$ under reducing conditions. ${ }^{57,58}$ When oxidized, these cysteines form two disulfide bonds and the $\mathrm{Zn}^{2+}$ is released from the protein. ${ }^{59} \mathrm{~A}$ second region on the $\mathrm{C}$-terminal domain of $\mathrm{Hsp} 33$ senses denaturing conditions. ${ }^{60}$ The combination of oxidizing and denaturing conditions triggers full activation and dimerization of $\mathrm{Hsp} 33$, which then acts as a holdase with broad specificity. ${ }^{55} \mathrm{Hsp} 33$ efficiently protects the cell from protein aggregation in the cytoplasm under aggregation inducing conditions. ${ }^{61}$

CsgA traverses the periplasmic space where it may be exposed to periplasmic proteostatic mediators that may play a role in maintaining CsgA in a soluble form prior to secretion across the outer membrane. The curli specific accessory protein CsgE exhibits chaperone activity toward CsgA in vitro by inhibiting polymerization. ${ }^{36}$ Here, we show that Spy, a newly characterized periplasmic holdase, ${ }^{62}$ can prevent $\mathrm{Csg} \mathrm{A}$ from polymerizing into an amyloid aggregate in vitro. Spy is under the regulation of the Cpx and Bae two-component systems, both of which are induced by stresses that promote protein misfolding and aggregation. ${ }^{63-66}$ Interestingly, both of the curli operons are negatively regulated by the Cpx system. ${ }^{65}$ Whether the Cpx stress response is induced during curli biogenesis has yet to be determined.

In this study we demonstrate that Hsp33, DnaK and Spy are potent inhibitors of CsgA fiber formation in vitro. Substoichiometric amounts of DnaK transiently prevent CsgA polymerization in the absence of the co-chaperones DnaJ and GrpE, and without the addition of ATP. Hsp33 not only interfered with polymerization initiation, but also reduced the total amount of amyloid fibers formed. Spy, in addition to inhibiting CsgA polymerization in vitro, reduced curli biogenesis when overexpressed in vivo. The inhibition of CsgA fiber formation by DnaK, Hsp33 and Spy can be overcome by the addition of preformed CsgA fibers. Moreover, we also found that Hsp33 and DnaK did not prevent the formation of a structurally conserved transient CsgA intermediate that is also formed by disease-associated amyloids. These chaperones may be part of a control mechanism protecting the cell from inappropriate amyloid formation within the cell.

\section{Results}

DnaK inhibits Csg $\widehat{A}$ amyloid formation. The conversion of soluble amyloidogenic monomeric proteins to an ordered amyloid fiber in vitro can be measured using the amyloid specific dye thioflavin T (ThT). When freshly purified CsgA was mixed with ThT, fluorescence emission increased rapidly after an approximately $2 \mathrm{~h}$ lag phase (Fig. 1A). The rapid growth phase of CsgA fibers was followed by a stationary phase, consistent with previous findings (Fig. 1A). ${ }^{7}$ When comparing CsgA polymerization profiles in the presence or absence of chaperone proteins, we measured two parameters. The first kinetic parameter was calculated as the time period preceding rapid fiber growth, called lag phase. The second parameter, called the elongation phase, was calculated as the time period encompassing the fiber growth phase from initiation of rapid polymerization to its completion. ${ }^{7,67}$

To determine if DnaK affects CsgA amyloid formation in vitro, ThT fluorescence was measured in the presence or absence of DnaK. DnaK increased the lag phase 3- and 8-fold at molar ratios of 1:20 and 1:5 (DnaK:CsgA), respectively (Fig. 1A). However, once CsgA started to aggregate, neither the rate of fiber formation nor the maximal ThT fluorescence were affected by the presence of the chaperone (Fig. 1A). To determine if the ability of DnaK to inhibit CsgA polymerization was dependent on the intact DnaK substrate-binding domain, the DnaK ${ }^{\mathrm{V} 436 \mathrm{~F}}$ mutant was tested in the ThT assay. The DnaK ${ }^{\mathrm{V} 436 \mathrm{~F}}$ mutant has reduced substrate affinity, but similar substrate specificity. ${ }^{68}$ When added to soluble CsgA, DnaK ${ }^{\mathrm{V} 436 \mathrm{~F}}$ was significantly less efficient at inhibiting CsgA fiber formation compared with wild-type DnaK 


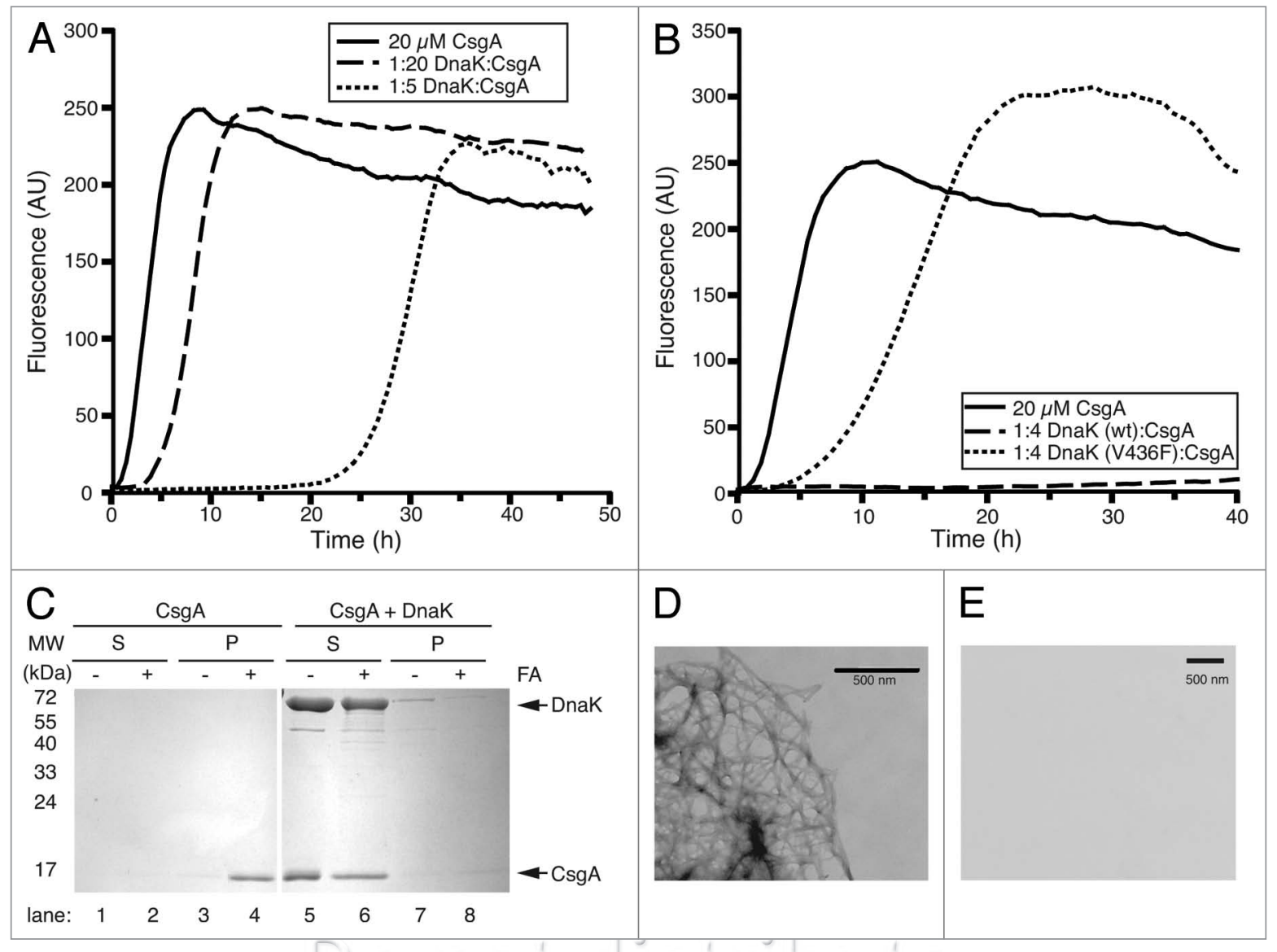

Figure 1. In vitro CsgA fiber formation in the presence of DnaK. (A) Freshly purified $20 \mu \mathrm{M}$ CsgA was incubated with $20 \mu \mathrm{M}$ ThT alone or with various concentrations of DnaK. Fluorescence was measured in $10 \mathrm{~min}$ intervals at $495 \mathrm{~nm}$ after excitation at $438 \mathrm{~nm}$. Fluorescence is reported in arbitrary units (AU). (B) A mutation in the substrate-binding domain significantly reduces DnaK's inhibitory effect on CsgA fiber formation in vitro. Freshly purified $20 \mu \mathrm{M}$ CsgA was incubated with $20 \mu \mathrm{M}$ ThT alone or with either DnaK or DnaK ${ }^{\mathrm{V} 336 \mathrm{~F}}$ at a 1:4 molar ratio (DnaK:CsgA). Fluorescence was measured as in (A). (A and B) are representative data of three replicates. (C) After $13 \mathrm{~h}$ samples were taken from the CsgA alone or DnaK + CsgA (1:4 molar ratio, DnaK:CsgA) polymerization reactions shown in (A) and separated into soluble (S) and polymerized (P) fractions by centrifugation prior to treatment with FA. The fractions were run on a 13\% SDS-PAGE gel and visualized by Coomassie brilliant blue staining. At the same timepoint $10 \mu \mathrm{l}$ samples containing CsgA only (D) or CsgA and DnaK (E) were applied to formvar coated copper grids, stained with uranyl actetate and examined by TEM.

(Fig. 1B). Wild-type DnaK prevented CsgA polymerization for greater than $30 \mathrm{~h}$, while an equivalent concentration of DnaK $\mathrm{V}^{\mathrm{V} 336 \mathrm{~F}}$ only increased the lag phase from 2-7 h (Fig. 1B).

To verify that the observed effect was due to the inhibition of CsgA polymerization, and not due to an indirect effect such as interference of the chaperone with ThT fluorescence we measured CsgA solubility in the presence and absence of DnaK. As an amyloid, curli are highly resistant to denaturation and require treatment with a harsh denaturant such as formic acid (FA) or hexafluoro-2-propanol (HFIP) to liberate the monomeric species. ${ }^{12,38} \mathrm{After} 13 \mathrm{~h}$ of incubation, polymerization reactions were centrifuged to separate the soluble and insoluble CsgA prior to analysis by SDS-PAGE. Duplicate samples were treated with $80 \%$ (w/w) FA to depolymerize CsgA aggregates. ${ }^{12,69}$ CsgA was present only in the FA treated pellet samples after $13 \mathrm{~h}$ of incubation in the absence of DnaK indicating that CsgA was entirely in the polymerized form (Fig. 1C and lanes 1-4). After $13 \mathrm{~h}$ in the presence of DnaK, however, CsgA was still detected in the non-FA treated supernatant samples suggesting that DnaK maintains CsgA in a soluble state (Fig. 1C and lanes 5-8). These results were in agreement with transmission electron micrograph (TEM) images. CsgA formed unbranched fibers in the absence of DnaK; however, no such fibers were observed when CsgA was incubated in the presence of DnaK for $13 \mathrm{~h}$ (Fig. 1D and E).

Oxidized Hsp33 inhibits CsgA polymerization in vitro. Hsp33 is a homodimeric molecular chaperone which, when activated by oxidative stress, functions as a potent holdase. To determine if $\mathrm{Hsp} 33$ influences amyloid formation, we measured CsgA fiber formation in vitro using ThT fluorescence. ${ }^{70,71}$ Reduced, chaperone-inactive, Hsp33red and oxidized, chaperone-active, Hsp33ox, were assayed for the ability to inhibit CsgA polymerization in vitro. ${ }^{55}$ The presence of Hsp33ox at a 1:80 (Hsp33ox dimer: $\mathrm{Csg} A$ ) molar ratio increased the lag phase of CsgA fiber formation to $8 \mathrm{~h}$ (Fig. 2A). The presence of Hsp33ox at a molar ratio of 1:20 increased the lag phase by $13 \mathrm{~h}$, while a molar ratio of $1: 4$ prevented CsgA polymerization for a time that was greater than the duration of the experiment (Fig. 2A). To verify that Hsp33 interfered with CsgA fiber formation and not with the 


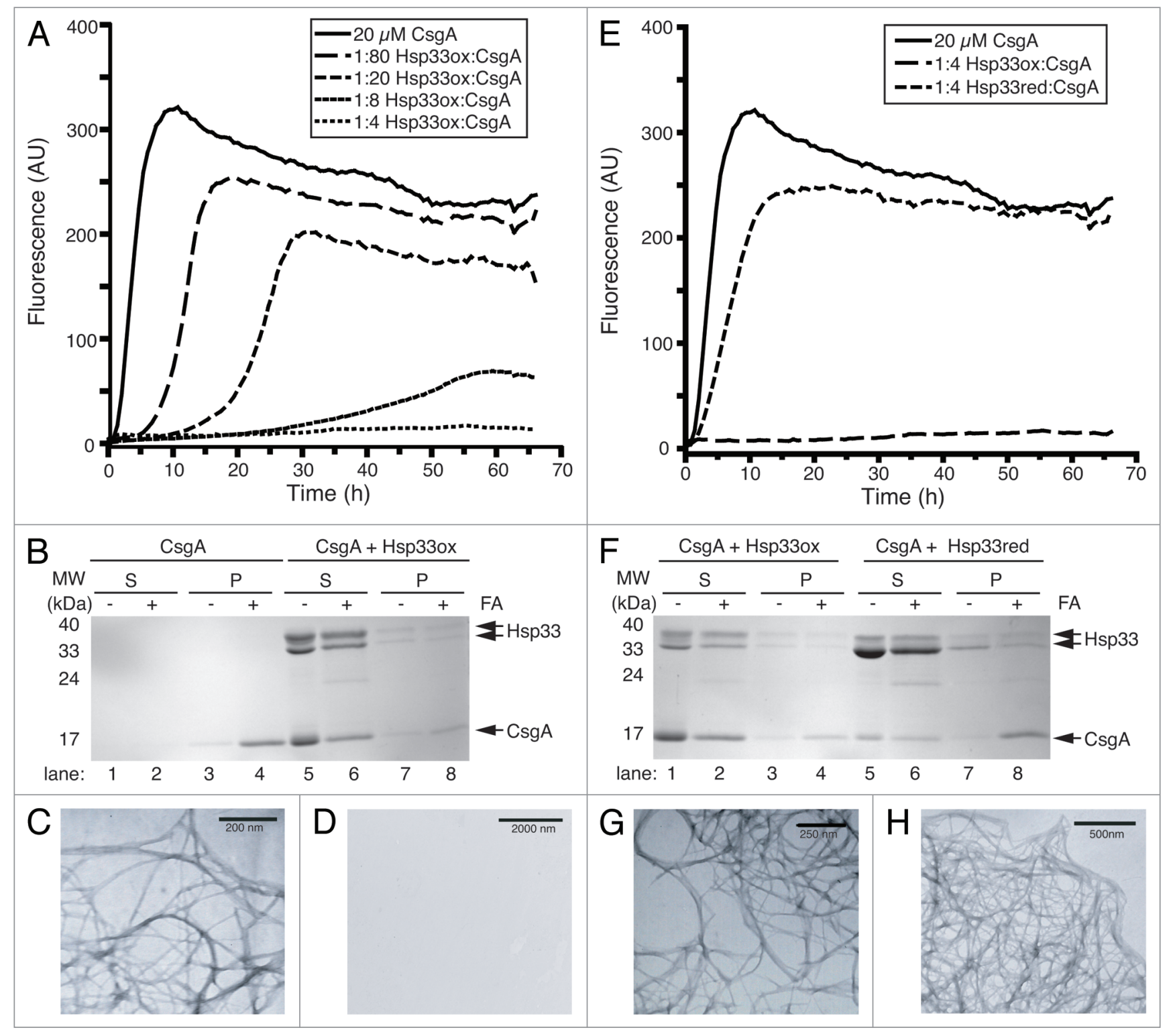

Figure 2. In vitro CsgA fiber formation in the presence of Hsp33. (A) Freshly purified $20 \mu \mathrm{M}$ CsgA was incubated with $20 \mu \mathrm{M}$ ThT both alone and with various concentrations of Hsp33ox. Fluorescence was measured as in Figure $1 \mathrm{~A}$. Shown are representative data of three replicates. (B) After $13 \mathrm{~h}$, samples were taken from the $\mathrm{CsgA}$ alone or $\mathrm{Hsp330x}+\mathrm{CsgA}$ at a 1:4 molar ratio (Hsp33:CsgA) polymerization reactions shown in (A) separated into soluble (S) and polymerized (P) fractions and treated as described in Figure 1C. At the same timepoint, $10 \mu$ l samples containing CsgA only (C) or CsgA and Hsp33ox (D) were applied to formvar coated copper grids, stained with uranyl actetate and examined by TEM. Hsp33red only minimally effects in vitro CsgA polymerization. (E) Freshly purified $20 \mu \mathrm{M} \mathrm{CsgA}$ was incubated with $20 \mu \mathrm{M}$ ThT alone or with either Hsp33ox or Hsp33red, each at a

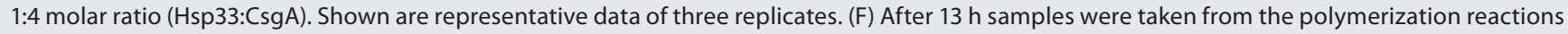
(1:4Hsp33ox:CsgA and 1:2 Hsp33red:CsgA), separated into soluble $(S)$ and polymerized $(P)$ fractions and treated as described in Figure 1C. Samples were also analyzed by negative stain TEM. CsgA only (G) or CsgA and Hsp33red (H) were applied to formvar coated copper grids, stained with uranyl acetate and examined by TEM.

ThT fluorescence, a CsgA solubility assay was conducted. All CsgA-containing samples had one band corresponding to CsgA in the pellet fraction treated with FA (Fig. 2B, lane 4). The majority of $\mathrm{Csg} A$ was present in the supernatant fraction of samples that contained Hsp33ox and CsgA at a 1:2 molar ratio signifying that CsgA was maintained in an SDS-soluble form (Fig. 2B, lanes 5-6). Fibers were observed by TEM in samples containing only CsgA, while no fibers were observed in samples containing both CsgA and Hsp33ox (Fig. 2C and D).
In contrast to Hsp33ox, Hsp33red had only marginal effects on CsgA polymerization in vitro (Fig. 2E). Consistent with the ThT results, CsgA was mostly present in the pellet fraction of samples containing Hsp33red and $\mathrm{CsgA}$ at a 1:2 molar ratio (Fig. 2F, lane 8). Significantly smaller amounts of CsgA were present in the supernatant fraction along with large quantities of Hsp33red (Fig. 2F, lanes 5-6). CsgA polymerization was also monitored by TEM. Regular, unbranched fibers were observed when CsgA was incubated alone or with Hsp33red for $13 \mathrm{~h}$ 


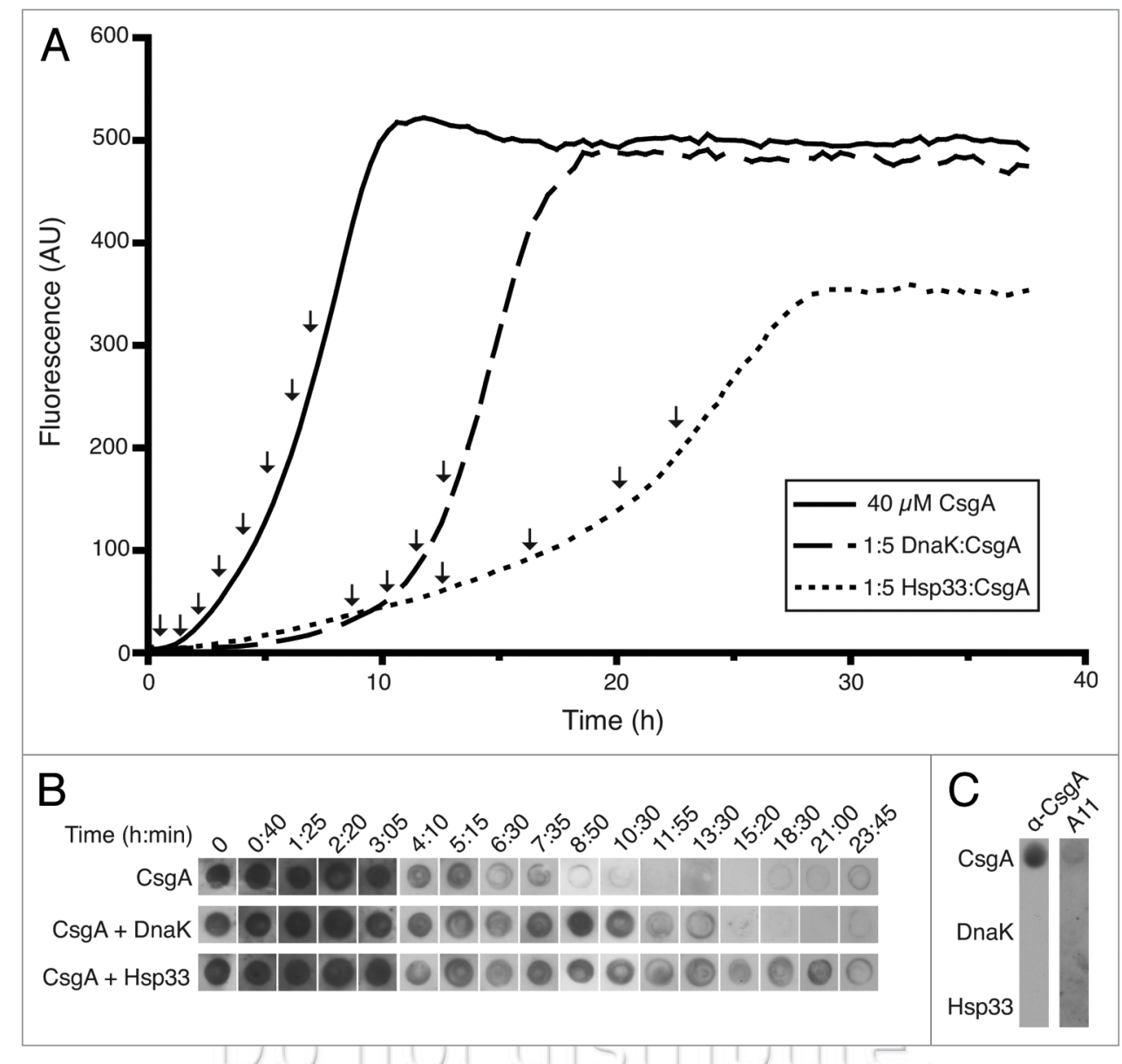

Figure 3. Hsp33ox and DnaK significantly prolong the presence of a CsgA intermediate that is recognized by the A11 antibody. (A) Freshly purified $40 \mu \mathrm{M}$ CsgA was incubated with $20 \mu \mathrm{M}$ ThT alone or with either DnaK or Hsp33ox. Fluorescence was measured as described in Figure 1A. Shown are representative data of three replicates. (B) Samples $(3 \mu \mathrm{l})$ of identical polymerization reactions, containing no ThT, were spotted on a nitrocellulose membrane and were probed with the A11 antibody. Samples were taken at indicated intervals (arrows) over the course of $24 \mathrm{~h}$. (C) Freshly purified 40 $\mu \mathrm{M}$ CsgA, $5 \mu \mathrm{M}$ DnaK and $5 \mu \mathrm{M} \mathrm{Hsp33ox}$, the same concentrations used in parts ( $\mathrm{A}$ and $\mathrm{B}$ ), were spotted on a nitrocellulose membrane and probed with the A11 antibody.

(Fig. 2G and $\mathrm{H}$ ). Together, these results demonstrate that oxidized, but not reduced, Hsp33 maintains CsgA in a soluble state.

Hsp33ox and DnaK prolong the presence of a transient folding intermediate common to amyloids. The conformationspecific antibody A11 recognizes a conserved, transient folding intermediate that is present during the polymerization of many functional and disease-associated amyloids. ${ }^{7,72,73}$ Although the exact molecular structure that A11 recognizes is unresolved, it is proposed to be an ordered intermediate that precedes the mature amyloid fiber. ${ }^{72}$ A11 transiently recognizes freshly purified CsgA during the first part of its transition to an amyloid fiber. However, once polymerization reaches stationary phase, no A11 reactive species can be observed. ${ }^{7}$ The A11 antibody was therefore used to test if Hsp33ox and DnaK act on CsgA in a manner that impacts progression from an A11-reactive conformation to an amyloid conformation. Freshly purified CsgA monomers were incubated with substoichiometric concentrations of DnaK or Hsp33ox. Samples were removed at the indicated time points (Fig. 3A, arrows) and spotted onto a nitrocellulose membrane. An A11-reactive species was detectable up to approximately $8 \mathrm{~h}$ when CsgA was incubated in the absence of chaperone (Fig. 3A and $B$ ). In the presence of DnaK, the lag phase is increased from $2 \mathrm{~h}$ to $10 \mathrm{~h}$ and the A11-reactive species was detectable for up to 15 h (Fig. 3A and B). When CsgA was incubated with Hsp33ox the A11-reactive species did not disappear for more than $24 \mathrm{~h}$ after the start of the reaction (Fig. 3B). The persistence of the A11 reactive species was not due to cross-reactivity with Hsp33ox, as the A11 antibody did not react with the above assayed concentration of purified Hsp33ox (Fig. 3C). The small amount of crossreactivity observed for the A11 antibody with DnaK (Fig. 3C) is consistent with previous findings that the A11 antibody reacts with Hsp70 and particularly with the substrate binding domain of Hsp70; however, Lotz et al. did not observe reactivity with full-length DnaK. ${ }^{45,74}$

The inhibitory effects of Hsp33ox and DnaK are abolished by the addition of CsgA seed. CsgA amyloid formation can be accelerated or "seeded" by the addition of preformed CsgA fibers.? To determine whether DnaK and Hsp33ox alter CsgA seeding by preformed fibers, polymerization assays were performed in the presence of different CsgA seed concentrations. CsgA seed 

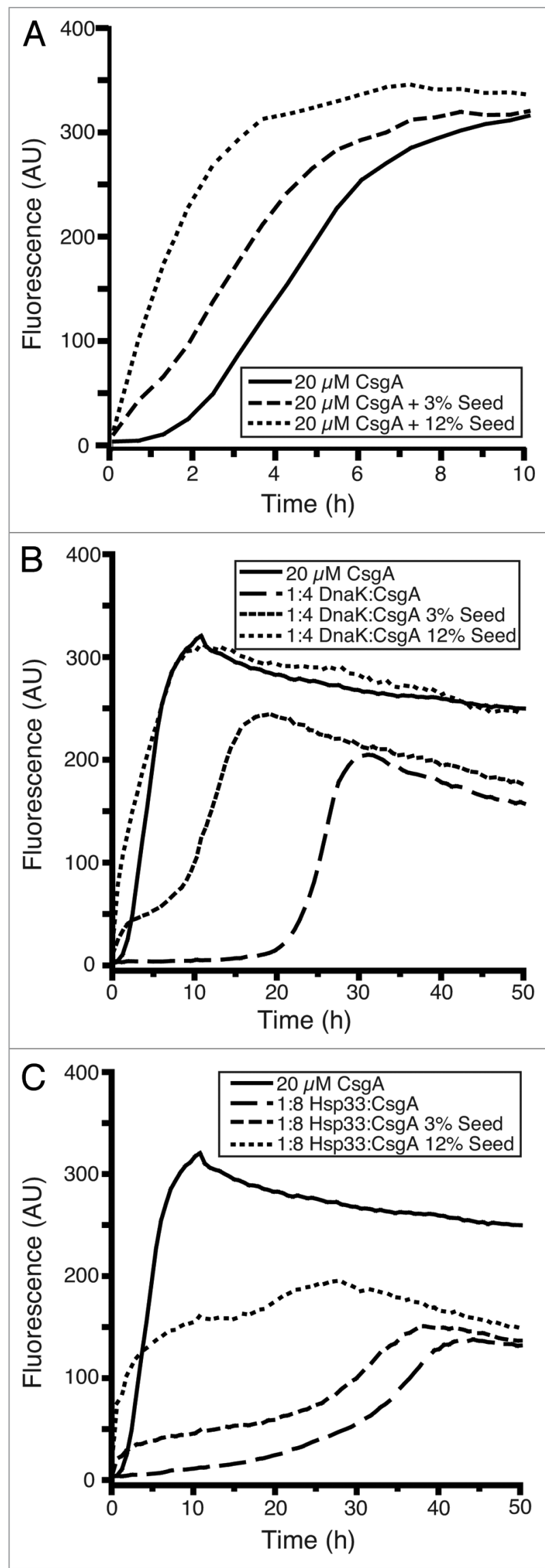

Figure 4. The addition of preformed fibers abolishes the inhibitory effects of Hsp33ox and DnaK on CsgA amyloid formation. (A) Freshly purified $20 \mu \mathrm{M}$ CsgA was incubated with $20 \mu \mathrm{M}$ ThT and DnaK (B) or Hsp33ox (C). In addition, 3\% (w/w relative to CsgA monomer) or $12 \%$ sonicated, preformed CsgA fibers were added to the polymerization reactions as indicated. Fluorescence was measured as described in Figure $1 \mathrm{~A}$. Shown are representative data sets of three replicates.

concentrations as low as 3\% (weight/weight relative to soluble monomer) eliminated the lag phase of CsgA amyloid formation as has been previously demonstrated (Fig. 4A). ${ }^{7}$ The inhibitory effects of DnaK and Hsp33ox, as well as the lag phase of CsgA fiber formation, were reduced by the addition of $3 \%(\mathrm{w} / \mathrm{w}) \mathrm{CsgA}$ seed and abolished by $12 \%(\mathrm{w} / \mathrm{w})$ CsgA seed for both chaperones (Fig. 4B and C; note the time scales are different in A from $\mathrm{B}$ and $\mathrm{C}$ ). In the presence of $3 \%$ seed there was an initial burst of fluorescence signal, a phase of minimal fluorescence increase and a more rapid growth phase that concluded in a stationary phase (Fig. 4B and C). Hsp33 reduced the maximum ThT fluorescence by a similar amount in the presence and absence of CsgA seed (Fig. 4C). The observation that the final ThT fluorescence remains reduced in the presence of $\mathrm{Hsp} 33$ and $12 \%$ CsgA seeds suggests that $\mathrm{Hsp} 33$ is sequestering a population of CsgA that cannot be incorporated into an amyloid (Fig. 4C, compare solid and dotted lines).

Spy inhibits CsgA polymerization in vitro. Spy is a periplasmic chaperone that is regulated by the Cpx and Bae extracellular stress responses, both of which are induced by conditions that stress the proteome. ${ }^{63-66}$ Spy exists as a homodimer and is structurally homologous to CpxP, the adaptor protein of the Cpx two-component stress response system. CpxP has also been proposed to act as a chaperone. ${ }^{62,75,76}$ However, Spy has two hydrophobic patches in the concave surface of its structure that may be involved in substrate binding and chaperone activity; similar hydrophobic patches are less well-defined in CpxP. ${ }^{62,76}$ We chose to test CsgA as a substrate for Spy since CsgA passes through the periplasm and, as a functional amyloid, is prone to ordered aggregation. No amyloid aggregates have been detected in the periplasm, therefore, we hypothesized that chaperone proteins like Spy may help to keep CsgA soluble as it passes through the periplasm to the cell surface.

To test the ability of Spy to prevent CsgA aggregation, we mixed purified Spy with freshly purified, monomeric CsgA and monitored polymerization in vitro by ThT fluorescence. Spy inhibited CsgA polymerization in a concentration dependent manner (Fig. 5A). Like DnaK and Hsp33, inhibition by Spy could also be overcome by the addition of preformed fibers (Fig. 5B). We found that Spy inhibited CsgA polymerization but the addition of comparable concentrations of $\mathrm{CpxP}$ to soluble CsgA had no effect on the polymerization of CsgA (data not shown). Comparison of these seemingly similar proteins will likely reveal their distinct functions in envelope stress responses.

Deletion of spy from the curli-expressing strain BW25113 had no effect on curli biogenesis (data not shown). It has been shown that some periplasmic chaperones are functionally redundant 


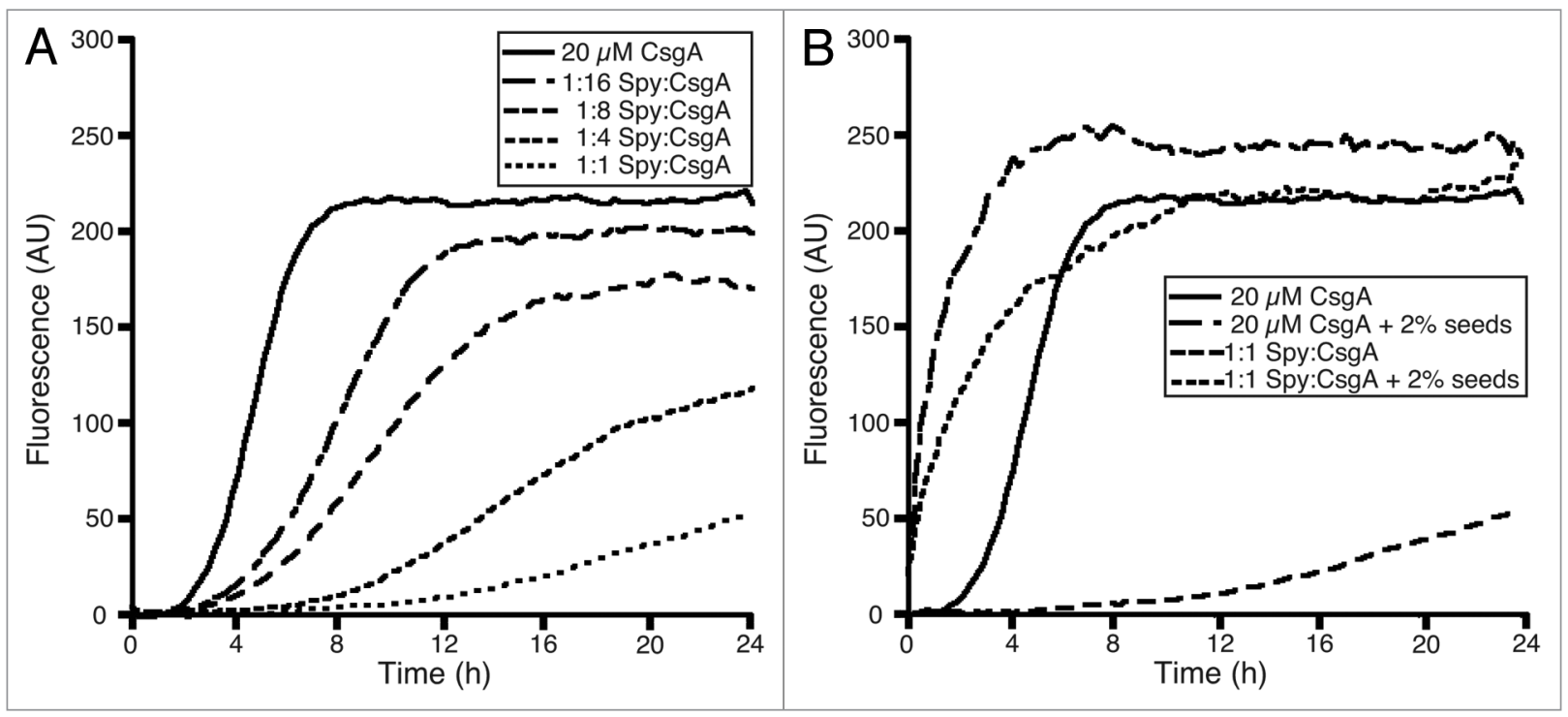

Figure 5. In vitro fiber formation in the presence of Spy. (A) $20 \mu \mathrm{M}$ freshly purified CsgA was incubated with $20 \mu \mathrm{M}$ ThT alone or in the presence of various molar ratios of Spy. Fluorescence was measured as described in Figure 1A. (B) Freshly purified $20 \mu \mathrm{M}$ CsgA was incubated with $20 \mu \mathrm{M}$ ThT alone or in the presence of $20 \mu \mathrm{M}$ Spy with and without $2 \%$ (w/w relative to CsgA monomer) sonicated, preformed CsgA fibers. Fluorescence was measured as described in Figure 1A. Shown are representative data sets of three replicates.

and can compensate for the loss of a single chaperone. ${ }^{77,78}$ It is therefore not surprising that the spy deletion did not produce an observable phenotype. However, when Spy was overexpressed, we observed reduced Congo red binding (Fig. 6A) consistent with reduced CsgA protein levels by protein gel blot (Fig. 6B). One possible explanation for this result is that stabilization of unfolded CsgA by Spy in the periplasm allows for more efficient proteolytic degradation of CsgA and therefore less secretion and curli assembly. Alternatively, the excessive production and secretion of Spy may have titrated resources that are required for the production and secretion of curli subunits. CsgG levels are not as dramatically reduced as CsgA levels, suggesting that CsgA is more sensitive to Spy overexpression. Because CsgG levels remain similar to WT when Spy is overexpressed, we concluded that there was not competition for secretion or significant feedback repression of the curli operons (Fig. 6B).

Next we tested whether reduced curli assembly was due to an increased chaperone capacity in the periplasm. Periplasmic protein extracts (PEs) were harvested and added back to freshly purified CsgA monomers, polymerization was monitored by ThT fluorescence in vitro (Fig. 6C). We harvested periplasmic proteins from WT BW25113 with empty vector (vector) or overexpressing Spy (pSpy). PEs from cells with empty vector showed a minimal effect on CsgA polymerization (Fig. 6C and long dashes). However, PEs from cells overexpressing Spy inhibited CsgA polymerization (Fig. 6C, short dashes). After $20 \mathrm{~h}$, CsgA was found to be in an SDS-insoluble state after incubation with periplasmic extracts from WT cells with empty vector only but primarily in an SDS-soluble state after incubation with periplasmic extracts from cells overexpressing Spy (Fig. 6D). These results demonstrate that the periplasmic chaperone activity was likely due to an increase in Spy protein in the periplasm.

\section{Discussion}

Amyloidogenesis is an ordered aggregation process. Amyloidogenic proteins, such as CsgA, are unstructured prior to their assembly into highly ordered aggregates making them likely targets for chaperone proteins. Consistent with this idea, human Hsp70 influences amyloid fiber formation of $A \beta(1-42)$ and $\alpha$-synuclein. ${ }^{43,44}$ Also, the small heat shock protein (sHsp) $\alpha \mathrm{B}$-crystallin and the sHsp-like clusterin have been shown to influence amyloid formation. ${ }^{79-81}$ Our results demonstrate that the endogenous bacterial chaperones DnaK, Hsp33 and Spy are potent inhibitors of CsgA fiber formation.

Like most amyloidogenic proteins, CsgA polymerizes by a nucleation-precipitation growth mechanism. Fiber growth is preceded by a lag phase, during which nuclei putatively form and subsequently drive fiber formation. Nucleation is the rate-limiting step of CsgA polymerization, resulting in an approximately 2 $\mathrm{h}$ lag phase (Fig. 4A) during which "needlelike" protofibers have been observed by TEM..$^{82,83}$ The presence of DnaK and Hsp33 significantly increased the length of the lag phase of CsgA polymerization, apparently by maintaining $\operatorname{Csg} \mathrm{A}$ in a soluble state. This data suggests that both chaperones interfere with CsgA amyloidogenesis. Spy also inhibited CsgA but required a 1:1 molar ratio (Spy dimer:CsgA monomer), suggesting either an alternative mechanism of inhibition or simply lower affinity for CsgA.

While DnaK increased the lag phase of CsgA polymerization, it does not appear to significantly affect fiber growth. The presence of DnaK did not appreciably change the time from the onset of fast polymerization to stationary phase or the maximum ThT fluorescence. The ability of DnaK to inhibit CsgA polymerization required an intact DnaK substrate-binding site, as the DnaK V436F mutant, which shows reduced substrate affinity, was far less efficient in inhibiting CsgA fiber formation. Therefore, we 


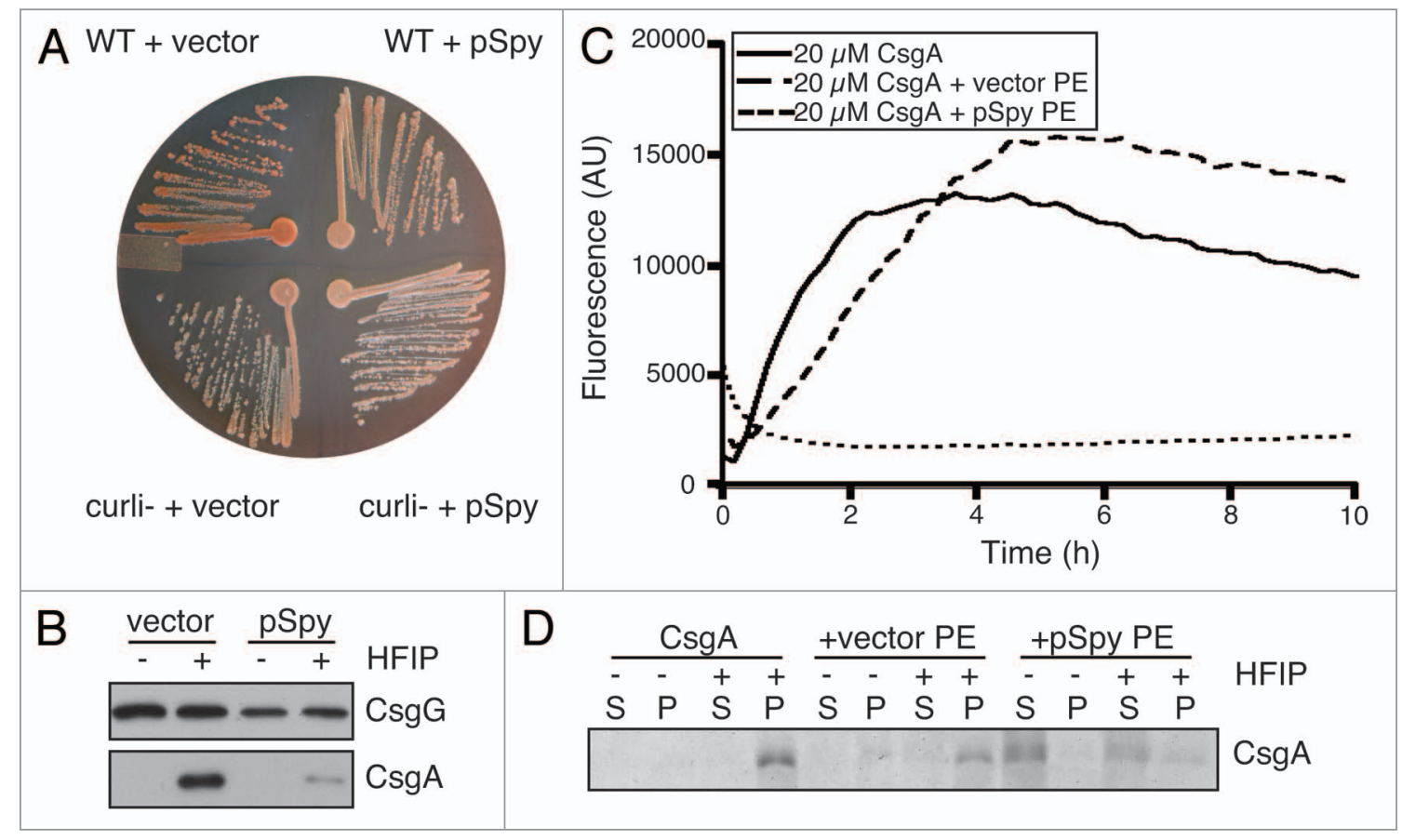

Figure 6. Overexpression of Spy reduces curli secretion and assembly. (A) WT BW25113 and curli- (csgBAC-csgDEFG-) were transformed with pCDFTrc (vector) or pCDFTrckanBamHI-spy (pSpy). Overnight cultures were washed with $50 \mathrm{mM} \mathrm{KPi} \mathrm{(pH} \mathrm{7.3)} \mathrm{and} \mathrm{diluted} \mathrm{to} \mathrm{OD}=1.0 .4 \mu \mathrm{L}$ of washed cells were spotted on a YESCA plate containing Congo red and grown for $2 \mathrm{~d}$ at $26^{\circ} \mathrm{C}$. (B) WT BW25113 cells transformed with vector control or pSpy were grown on a Congo red containing YESCA plate for $2 \mathrm{~d}$ at $26^{\circ} \mathrm{C}$. Cells were harvested, normalized by $\mathrm{OD}_{600}$ and subjected to protein gel blot using polyclonal antibodies against CsgG (top) and CsgA (bottom) (C) $20 \mu \mathrm{M}$ CsgA polymerization was monitored by ThT fluorescence in the presence of periplasmic extracts (PEs) from BW25113 + vector control or BW25113 + pSpy. PEs were normalized to $100 \mathrm{ug} / \mathrm{mL}$. Fluorescence was measured as described in Figure 1A. Shown are representative data of three replicates. (D) Soluble and insoluble CsgA were separated by centrifugation after incubation alone, with BW25113 + vector control or with BW25113 + pSpy periplasmic extracts. Samples were either treated with SDS loading buffer or first with hexafluoro-2-propanol (HFIP), dried and then treated with SDS loading buffer. Samples were loaded on an 18\% SDS PAGE gel and stained with Coomassie.

suggest that DnaK most likely interacts transiently with CsgA, preventing an early step in fiber formation, while it does not interfere with polymerization after nucleation in the absence of ATP. This model is also supported by the observation that the same maximal ThT fluorescence is detected in the presence and absence of DnaK.

In contrast to DnaK, Hsp33 not only lengthened the lag phase of CsgA fiber formation but it also altered CsgA fiber elongation kinetics. Hsp33 decreased the rate of elongation and reduced the total amount of polymerized CsgA. Fiber formation is prevented entirely at an Hsp33 to CsgA molar ratio of 1:4. This observation is similar to the results of previous studies with the sHsp-like clusterin. ${ }^{79}$ The inhibitory effect of Hsp33 is dependent on its redox state. The reduced form shows little inhibitory activity, while the oxidized form is a very potent inhibitor. We propose that Hsp33, like DnaK, binds to a folding intermediate, preventing nucleus formation or maturation from the "needlelike" oligomers into a fiber-competent conformation. Two models can be proposed in order to explain why sub-stoichiometric amounts of Hsp33 are sufficient to prevent CsgA fiber formation. Hsp33 could tightly associate with an oligomeric folding intermediate, arresting a large amount of CsgA in an elongation incompetent state, leading to a reduced CsgA monomer and amyloid fiber concentration. Such a reduced monomer concentration would explain the slower polymerization and overall reduced yield of amyloid fibers formed. Alternatively, a transient interaction could lead to the release of an altered CsgA molecule with modified amyloid forming potential. Similar models have been proposed to explain the inhibition of $\mathrm{A} \beta$ fiber formation by $\mathrm{Hsp} 70 / 40$ and Hsp90.43

Spy may inhibit CsgA polymerization at a step prior to oligomerization. Spy has a slight effect on CsgA polymerization at substoichiometric concentrations, but does not exhibit full inhibition unless at a 1:1 molar ratio of CsgA to Spy dimer (Fig. 5A). Seeding assays provide further evidence that Spy inhibits oligomerization given that the polymerization kinetics of a seeded reaction in the presence of Spy more closely resemble that of a seeded reaction in the absence of chaperone (Fig. 5B). These results suggest that Spy is stabilizing the soluble monomeric species of CsgA and are consistent with the findings of Quan et al. demonstrating full aggregation inhibition of denatured $\mathrm{MDH}$ and aldolase at a 1:1 molar ratio. ${ }^{62}$

It has been proposed that there are two nucleation pathways involved in amyloid fiber formation: (1) Fiber independent nucleation is involved in the initial formation of amyloid fibers during the lag phase and (2) fiber dependent nucleation predominates after initial fiber formation and is the driving force of seeded polymerization reactions. The presence of both pathways has been demonstrated for amyloid formation by Islet Amyloid Polypeptide. ${ }^{84}$ When CsgA monomer is nucleated by preformed fibers, the presence of Hsp33, DnaK or Spy no longer prevented 
the seeding of CsgA into an amyloid fiber (Figs. 4 and 5), suggesting that they interfere with fiber independent nucleation and not with fiber dependent nucleation. The folding step that is inhibited by Hsp33, DnaK and Spy might not be necessary for CsgA to be competent for fiber dependent nucleation. This would explain the observation that preformed fibers circumvent the inhibitory effects of these chaperones. However, even in a seeded reaction, $\mathrm{Hsp} 33$ reduces the amount of CsgA fibers formed, although no effect on the lag phase is observed. This observation suggests again that $\mathrm{Hsp} 33$ arrests a fraction of CsgA in a state incompetent for fiber formation. DnaK and Spy, on the other hand, appear to act at an earlier stage: possibly stabilizing soluble monomers of CsgA prior to oligomerization.

Disease associated amyloids such as $A \beta$ and $\operatorname{PrP}$ form a transient oligomeric species, which is recognized by the A11 antibody. ${ }^{72}$ Freshly purified CsgA is recognized by the A11 antibody, while $\operatorname{Csg} A$ denatured with guanidine hydrochloride is not recognized. ${ }^{7}$ The CsgA species that is recognized by the A11antibody has been shown to be either monomeric or dimeric, but may be an off-pathway step in amyloid fiber formation. ${ }^{7}$ DnaK and $\mathrm{Hsp} 33$ increase the time that the A11-recognized species is present during CsgA polymerization, suggesting that CsgA undergoes at least one rapid folding step into the A11-recognized species before the chaperones inhibit further folding and fiber elongation. Further experiments-will have to be performed to determine the relevance of the species recognized by the Al1antibody in the amyloid formation pathway of CsgA. Hsp33 may be a suitable tool for these experiments.

A role for DnaK, Hsp33 and Spy during curli biogenesis in vivo remains to be determined. Our data suggest that chaperones could provide a cellular control mechanism to protect the cell from premature amyloid formation prior to export of CsgA covering different physiological situations and in different subcellular compartments. They most likely interfere with fiber independent nucleation, which would be predominant in the cytosol after CsgA synthesis and during secretion across the periplasmic space. Curli assembly was reduced by increasing the chaperone capacity of the periplasm through the overexpression of Spy, suggesting that cells carefully balance curli subunit secretion with proteostatic mediators in the periplasm. Curli biogenesis is tightly controlled at the level of transcription, export and nucleation (reviewed in ref. 85); chaperone mediated assembly of curli adds another dimension to the controlled nature this system.

\section{Materials and Methods}

CsgA purification. CsgG and CsgA-6XHis were overexpressed in LSR12 (C600:: $\Delta c s g)$ and CsgA-6x His was purified from the growth media as previously described in references 7 and 12 . To remove the imidazole, eluates were run over a gel filtration column (Sephadex G-25 fine, Amersham Biosciences) into either 50 $\mathrm{mM}$ potassium phosphate buffer $\mathrm{pH} 7.2(\mathrm{KPi})$ or $50 \mathrm{mM} \mathrm{KPi}$ $\mathrm{pH} 7.2$ that was supplemented with $50 \mathrm{mM}$ potassium chloride $(\mathrm{KCl})$. CsgA-6x His polymerizes into an amyloid fiber with similar kinetics to wild-type $\operatorname{Csg} A$ in vitro and will be referred to as "CsgA" in this paper. ${ }^{12}$
Hsp33 purification. Hsp33 was purified as previously described in reference 55 . The reduction of $\mathrm{Hsp} 33$ was performed as previously described in reference 59. To oxidize $\mathrm{Hsp} 33$, it was treated with $2 \mathrm{mM} \mathrm{H}_{2} \mathrm{O}_{2}$ at $43^{\circ} \mathrm{C}$ for $1 \mathrm{~h}$ prior to buffer exchange into $40 \mathrm{mM} \mathrm{KPi} \mathrm{pH} \mathrm{7.5,} \mathrm{as} \mathrm{previously} \mathrm{described} \mathrm{in} \mathrm{reference} 60$.

DnaK purification. Wild-type and mutant DnaK (V436F) was purified as previously described in reference 86 with slight modifications. Mutant DnaK was created by site-directed mutagenesis using the QuikChange kit (Stratagene).

Spy purification. Spy was purified as previously described in reference 62.

Periplasmic extract preparation. WT BW25113 cells were made chemically competent and transformed with pCDFTrc (vector) or pCDFTrckanBamHI-spy $(\mathrm{pSpy})^{62}$ were grown in $\mathrm{LB}$ at $37^{\circ} \mathrm{C}$ overnight and then plated on YESCA plates supplemented with kanamycin and grown for $2 \mathrm{~d}$ at $26^{\circ} \mathrm{C}$. It was unnecessary to induce Spy expression as increased Spy levels could be observed by coomassie stained SDS-PAGE (data not shown). Cells were harvested in $20 \mathrm{mM}$ Tris ( $\mathrm{pH}$ 8.0). Cells were collected by centrifugation at $8,000 \mathrm{rcf}$ for $10 \mathrm{~min}$ followed by incubation in osmotic shock buffer $(30 \mathrm{mM}$ Tris, $40 \%$ sucrose 2 mM EDTA, pH 7.3) for 10 min. Cells were pelleted at room temperature and resuspended in $2 \mathrm{mM} \mathrm{MgCl}$, incubated on ice for $3 \mathrm{~min}$ and pelleted again. The supernatant was kept as the periplasmic extract (PE).

ThT fiber formation assay. Each experiment shown is a representative of at least three replicates. Freshly purified CsgA was mixed with $20 \mu \mathrm{M}$ ThT and incubated in the presence of DnaK or Hsp33 in a black 96-well flat bottom plate (Corning Inc.), which was placed in a Spectramax M2 plate reader (Molecular Devices; Figs. 1-4). The plate reader was maintained at $25^{\circ} \mathrm{C}$ for all experiments. $50 \mathrm{mM} \mathrm{KPi} \mathrm{pH} 7.2$ was used as reaction buffer for all experiments involving Hsp33. For experiments involving DnaK, $50 \mathrm{mM} \mathrm{KPi} 50 \mathrm{mM} \mathrm{KCl} \mathrm{pH} 7.2$ was used as reaction buffer. Unless stated differently the reaction volumes were $100 \mu \mathrm{l}$. Molar ratios are reported in terms of monomeric DnaK and dimeric Hsp33. Fluorescence was monitored in 10 min intervals at $495 \mathrm{~nm}$ after excitation at $438 \mathrm{~nm}$. The cut off of the plate reader was set to $475 \mathrm{~nm}$. The plate was shaken for $5 \mathrm{sec}$ prior to each read. CsgA polymerization in the presence of Spy and periplasmic extracts were conducted in $50 \mathrm{uL}$ reaction volumes in 50 $\mathrm{mM} \mathrm{KPi} \mathrm{(pH} \mathrm{7.3)} \mathrm{with} 20 \mathrm{uM} \mathrm{ThT}$. ThT readings were taken in a Infinite 200 Pro NanoQuant plate reader (Tecan Group LTD.; Figs. 5 and 6) every $10 \mathrm{~min}$ after $2 \mathrm{sec}$ of shaking at $2.5 \mathrm{~mm}$ amplitude with an excitation wavelength of $438 \mathrm{~nm}$ and emission wavelength of $495 \mathrm{~nm}$. Molar ratios are reported in terms of Spy dimer. At least three replicates of each assay were conducted.

CsgA solubility assay. $100 \mu \mathrm{l}$ CsgA samples supplemented with the respective chaperones were incubated in 96-well plates in a Spectramax M2 plate reader at $25^{\circ} \mathrm{C}$. Identical samples were supplemented with $20 \mu \mathrm{M}$ ThT to monitor amyloid formation. ThT fluorescence was monitored as described above. After $13 \mathrm{~h} 30 \mu \mathrm{l}$ aliquots were taken from the CsgA samples and centrifuged at $70,000 \mathrm{xg}$ for $1 \mathrm{~h}$ at $4^{\circ} \mathrm{C}$ in $1.5 \mathrm{ml}$ polyallomer tubes (Beckman Instruments) using a TLA-55 rotor (Beckman Coulter) in an Optima TLX ultracentrifuge (Beckman Coulter). 
After centrifugation, the supernatant fractions $(-30 \mu l)$ were transferred into a fresh tube. Pellet fractions were treated with $50 \mu \mathrm{l} 95 \%$ (w/w) formic acid (Sigma-Aldrich, F0507), while 95\% formic acid was added to the supernatant fraction yielding a final formic acid concentration of $76 \%$. Immediately after addition of formic acid the samples were placed in a SPD111V SpeedVac (Thermo Fisher Scientific) and all solvent was removed. The formic acid treated samples were resuspended in 4x SDS sample buffer (125 mM Tris pH 6.8, 10\% 2-Mercaptoethanol, 20\% Glycerol, 6\% sodium dodecyl sulfate, $0.02 \%$ bromophenol blue), sonicated for ten minutes in a Solidstate/Ultrasonic FS-14 bath sonicator (Fisher Scientific) before incubating at $95^{\circ} \mathrm{C}$ for $10 \mathrm{~min}$. Formic acid treated and untreated samples were then analyzed by SDS-PAGE and Coomassie staining. The solubility of CsgA after incubation with periplasmic extracts was determined similarly. Soluble and insoluble CsgA were separated by centrifugation in an Eppendorf 5415R centrifuge in duplicate. Pellets were treated with $40 \mu \mathrm{L} 4 \mathrm{x}$ SDS sample buffer or $50 \mu \mathrm{L}$ hexafluoro-2-propanol (HFIP; Sigma Aldrich) for $10 \mathrm{~min}$ and then dried in a SPD111V SpeedVac (Thermo Fisher Scientific) before resuspension in $40 \mu \mathrm{L}$ of $4 \mathrm{x}$ SDS sample buffer. Supernatants were treated with and equal volume of $4 \mathrm{x}$ SDS sample buffer or with a final concentration of $80 \%$ HFIP for $10 \mathrm{~min}$, dried and then resuspended in $40 \mu \mathrm{L} 4 \mathrm{x}$ SDS sample buffer. Samples were boiled for $10 \mathrm{~min}$ prior to loading on a $15 \%$ SDS-PAGE gel and analyzed by Coomassie staining.

Dot-Blot Assay with A11. The blot assay was performed as previously described in reference 7 . CsgA samples were taken from fiber formation reactions in 96-well plates that were simultaneously monitored with ThT fluorescence in separate wells at $25^{\circ} \mathrm{C}$, as described above.

\section{References}

1. Chiti F, Dobson CM. Protein misfolding, functional amyloid and human disease. Annu Rev Biochem 2006; 75:333-66; PMID:16756495; http://dx.doi org/10.1146/annurev.biochem.75.101304.123901.

2. Westermark P. Quantitative studies on amyloid in the islets of Langerhans. Ups J Med Sci 1972; 77:91-4; PMID:4116019.

3. Kitamoto T, Hikita K, Tashima T, Tateishi J, Sato Y. Scrapie-associated fibrils (SAF) purification method yields amyloid proteins from systemic and cerebral amyloidosis. Biosci Rep 1986; 6:459-65; PMID:2874846; http://dx.doi.org/10.1007/BF01116137.

4. Klunk WE, Pettegrew JW, Abraham DJ. Quantitative evaluation of congo red binding to amyloid-like proteins with a beta-pleated sheet conformation. J Histochem Cytochem 1989; 37:1273-81; PMID:2666510; http:// dx.doi.org/10.1177/37.8.2666510.

5. Glover JR, Kowal AS, Schirmer EC, Patino MM, Liu JJ, Lindquist S. Self-seeded fibers formed by Sup35, the protein determinant of $\left[\mathrm{PSI}^{+}\right]$, a heritable prion-like factor of S. cerevisiae. Cell 1997; 89:811-9; PMID:9182769; http://dx.doi.org/10.1016/S00928674(00)80264-0.

6. Guijarro JI, Sunde M, Jones JA, Campbell ID, Dobson CM. Amyloid fibril formation by an SH3 domain. Proc Natl Acad Sci USA 1998; 95:4224-8; PMID:9539718; http://dx.doi.org/10.1073/pnas.95.8.4224.

7. Wang X, Smith DR, Jones JW, Chapman MR. In vitro polymerization of a functional Escherichia coli amyloid protein. J Biol Chem 2007; 282:3713-9; PMID:17164238; http://dx.doi.org/10.1074/jbc. M609228200.
Whole cell protein gel blot. BW25113 with pCDFT (vector) or pCDFTrckanBamHI-spy (pSpy) were grown overnight in LB supplemented with kanamycin at $37^{\circ} \mathrm{C}$. Cultures were diluted to $\mathrm{OD}=1.0$ in $50 \mathrm{mM} \mathrm{KPi}(\mathrm{pH} \mathrm{7.3})$ and $4 \mu \mathrm{L}$ were spotted on a YESCA plate and grown for $48 \mathrm{~h}$ at $26^{\circ} \mathrm{C}$. Cells were harvested in $1 \mathrm{~mL}$ of $50 \mathrm{mM} \mathrm{KPi}(\mathrm{pH} \mathrm{7.3)}$. Two $100 \mu \mathrm{L}$ samples were taken from a $1.0 \mathrm{OD}$ suspension and pelleted at 16,000 rcf for $10 \mathrm{~min}$. Samples were either treated with $40 \mu \mathrm{L}$ of $2 \mathrm{x}$ SDS loading buffer or $50 \mu \mathrm{L}$ of hexafluoro-2-propanol (Sigma-Aldrich, 52512), dried in a Thermo Savant SPD SpeedVac (Thermo Fisher Scientific) and then treated with $40 \mu \mathrm{L}$ of $2 x$ SDS loading buffer. Seven microliters were loaded in a $15 \%$ SDS PAGE gel, transferred to $0.45 \mu \mathrm{m}$ PVDF transfer membrane (Thermo Fisher Scientific) and blocked overnight in TBS-T 5\% skim milk. Blots were probed with anti-CsgA $(1: 10,000)$ and anti-CsgG $(1: 70,000)$ polyclonal antibodies followed by goat-anti-rabbit (1:10,000; Sigma-Aldrich, A0545).

Electron microscopy. Electron microscopy sample preparation and image acquisition was performed as previously described in reference 7 .

\section{Disclosure of Potential Conflicts of Interest}

No potential conflicts of interest were disclosed.

\section{( T) Acknowledgments}

We would like to thank the members of the Chapman lab for helpful discussions and review of this manuscript. This work was supported by NIH grants RO1 A1073847 and GM065318, by NIH NRSA T32 GM008353 and by the Intramural Research Program of the National Institutes of Health, National Cancer Institute, Center for Cancer Research.
8. Fändrich M, Fletcher MA, Dobson CM. Amyloid fibrils from muscle myoglobin. Nature 2001; 410:165-6; PMID:11242064; http://dx.doi. org/10.1038/35065514.

9. Epstein EA, Chapman MR. Polymerizing the fibre between bacteria and host cells: the biogenesis of functional amyloid fibres. Cell Microbiol 2008; 10:1413 20; PMID:18373633; http://dx.doi.org/10.1111/ j.1462-5822.2008.01148.x.

10. Hammer ND, Wang X, McGuffie BA, Chapman MR. Amyloids: friend or foe? J Alzheimers Dis 2008; 13:407-19; PMID:18487849.

11. Bieler S, Estrada L, Lagos R, Baeza M, Castilla J, Soto C. Amyloid formation modulates the biological activity of a bacterial protein. J Biol Chem 2005; 280:268805; PMID:15917245; http://dx.doi.org/10.1074/jbc. M502031200.

12. Chapman MR, Robinson LS, Pinkner JS, Roth R, Heuser J, Hammar M, et al. Role of Escherichia coli curli operons in directing amyloid fiber formation. Science 2002; 295:851-5; PMID:11823641; http:// dx.doi.org/10.1126/science.1067484.

13. Elliot MA, Karoonuthaisiri N, Huang J, Bibb MJ, Cohen SN, Kao CM, et al. The chaplins: a family of hydrophobic cell-surface proteins involved in aerial mycelium formation in Streptomyces coelicolor. Genes Dev 2003; 17:1727-40; PMID:12832397; http:// dx.doi.org/10.1101/gad.264403.

14. Claessen D, Rink R, de Jong W, Siebring J, de Vreugd P, Boersma FG, et al. A novel class of secreted hydrophobic proteins is involved in aerial hyphae formation in Streptomyces coelicolor by forming amyloid-like fibrils. Genes Dev 2003; 17:1714-26; PMID:12832396; http://dx.doi.org/10.1101/gad.264303.
15. Wickner RB. [URE3] as an altered URE2 protein: evidence for a prion analog in Saccharomyces cerevisiae. Science 1994; 264:566-9; PMID:7909170; http:// dx.doi.org/10.1126/science.7909170.

16. Coustou-Linares V, Maddelein ML, Begueret J, Saupe SJ. In vivo aggregation of the HET-s prion protein of the fungus Podospora anserina. Mol Microbiol 2001; 42:1325-35; PMID:11886562; http://dx.doi. org/10.1046/j.1365-2958.2001.02707.x.

17. True HL, Lindquist SL. A yeast prion provides a mechanism for genetic variation and phenotypic diversity. Nature 2000; 407:477-83; PMID:11028992; http:// dx.doi.org/10.1038/35035005.

18. Fowler DM, Koulov AV, Alory-Jost C, Marks MS, Balch WE, Kelly JW. Functional amyloid formation within mammalian tissue. PLoS Biol 2006; 4:6; PMID:16300414; http://dx.doi.org/10.1371/journal. pbio.0040006.

19. Maji SK, Perrin MH, Sawaya MR, Jessberger S, Vadodaria K, Rissman RA, et al. Functional amyloids as natural storage of peptide hormones in pituitary secretory granules. Science 2009; 325:328-32; PMID:19541956; http://dx.doi.org/10.1126/science. 1173155 .

20. Austin JW, Sanders G, Kay WW, Collinson SK. Thin aggregative fimbriae enhance Salmonella enteritidis biofilm formation. FEMS Microbiol Lett 1998; 162:295-301; PMID:9627964; http://dx.doi. org/10.1111/j.1574-6968.1998.tb13012.x. 
21. Zogaj X, Bokranz W, Nimtz M, Romling U. Production of cellulose and curli fimbriae by members of the family Enterobacteriaceae isolated from the human gastrointestinal tract. Infect Immun 2003; 71:41518; PMID:12819107; http://dx.doi.org/10.1128/ IAI.71.7.4151-8.2003

22. Zogaj X, Nimtz M, Rohde M, Bokranz W, Romling U. The multicellular morphotypes of Salmonella typhimurium and Escherichia coli produce cellulose as the second component of the extracellular matrix. Mol Microbiol 2001; 39:1452-63; PMID:11260463; http://dx.doi.org/10.1046/j.1365-2958.2001.02337.x.

23. Johansson C, Nilsson T, Olsen A, Wick MJ. The influence of curli, a MHC-I-binding bacterial surface structure, on macrophage- $T$ cell interactions. FEMS Immunol Med Microbiol 2001; 30:21-9; PMID:11172987.

24. Gophna U, Barlev M, Seijffers R, Oelschlager TA, Hacker J, Ron EZ. Curli fibers mediate internalization of Escherichia coli by eukaryotic cells. Infect Immun 2001; 69:2659-65; PMID:11254632; http://dx.doi. org/10.1128/IAI.69.4.2659-65.2001.

25. Gophna U, Oelschlaeger TA, Hacker J, Ron EZ. Role of fibronectin in curli-mediated internalization. FEMS Microbiol Lett 2002; 212:55-8; PMID:12076787; http://dx.doi.org/10.1111/j.1574-6968.2002. tb11244.x.

26. Bian Z, Brauner A, Li Y, Normark S. Expression of and cytokine activation by Escherichia coli curli fibers in human sepsis. J Infect Dis 2000; 181:602-12; PMID:10669344; http://dx.doi.org/10.1086/315233.

27. Bian Z, Yan ZQ, Hansson GK, Thoren P, Normark S. Activation of inducible nitric oxide synthase/nitric oxide by curli fibers leads to a fall in blood pressure during systemic Escherichia coli infection in mice. I Infect Dis 2001; 183:612-9; PMID:1 1170987; http://dx.doi. org/10.1086/318528.

28. Tükel C, Raffatellu M, Humphries AD, Wilson RP, Andrews-Polymenis HL, Gull T, et al. CsgA is a pathogen-associated molecular pattern of Salmonella enterica serotype Typhimurium that is recognized by Toll-like receptor 2. Mol Microbiol 2005; 58:289-304; PMID:16164566; http://dx.doi.org/10.1111/j.13652958.2005.04825.x.

29. Hammar M, Arnqvist A, Bian Z, Olsen A, Normark $S$. Expression of two csg operons is required for production of fibronectin- and congo red-binding curli polymers in Escherichia coli K-12. Mol Microbiol 1995; 18:661-70; PMID:8817489; http://dx.doi. org/10.1111/j.1365-2958.1995.mmi_18040661.x.

30. Loferer H, Hammar M, Normark S. Availability of the fibre subunit CsgA and the nucleator protein $\mathrm{Csg} \mathrm{B}$ during assembly of fibronectin-binding curli is limited by the intracellular concentration of the novel lipoprotein CsgG. Mol Microbiol 1997; 26:11-23; PMID:9383186; http://dx.doi.org/10.1046/j.13652958.1997.5231883.x.

31. Robinson LS, Ashman EM, Hultgren SJ, Chapman MR. Secretion of curli fibre subunits is mediated by the outer membrane-localized CsgG protein. Mol Microbiol 2006; 59:870-81; PMID:16420357; http:// dx.doi.org/10.1111/j.1365-2958.2005.04997.x.

32. Bian Z, Normark S. Nucleator function of CsgB for the assembly of adhesive surface organelles in Escherichia coli. EMBO J 1997; 16:5827-36; PMID:9312041; http://dx.doi.org/10.1093/emboj/16.19.5827.

33. Hammar M, Bian Z, Normark S. Nucleatordependent intercellular assembly of adhesive curli organelles in Escherichia coli. Proc Natl Acad Sci USA 1996; 93:6562-6; PMID:8692856; http://dx.doi. org/10.1073/pnas.93.13.6562.

34. Hammer ND, Schmidt JC, Chapman MR. The curli nucleator protein, $\mathrm{CsgB}$, contains an amyloidogenic domain that directs CsgA polymerization. Proc Natl Acad Sci USA 2007; 104:12494-9; PMID:17636121; http://dx.doi.org/10.1073/pnas.0703310104.
35. Nenninger AA, Robinson LS, Hultgren SJ. Localized and efficient curli nucleation requires the chaperonelike amyloid assembly protein $\mathrm{CsgF}$. Proc Natl Acad Sci USA 2009; 106:900-5; PMID:19131513; http:// dx.doi.org/10.1073/pnas.0812143106.

36. Nenninger AA, Robinson LS, Hammer ND, Epstein EA, Badtke MP, Hultgren SJ, et al. CsgE is a curli secretion specificity factor that prevents amyloid fibre aggregation. Mol Microbiol 2011; 81:486-99; PMID:21645131; http://dx.doi.org/10.1111/j.13652958.2011.07706.x

37. Gibson DL, White AP, Rajotte CM, Kay WW. AgfC and AgfE facilitate extracellular thin aggregative fim briae synthesis in Salmonella enteritidis. Microbiology 2007; 153:1131-40; PMID:17379722; http://dx.doi. org/10.1099/mic.0.2006/000935-0.

38. Taylor JD, Zhou Y, Salgado PS, Patwardhan A, McGuffie M, Pape T, et al. Atomic Resolution Insights into Curli Fibre Biogenesis. Structure 2011; 19:130716; PMID:21893289; http://dx.doi.org/10.1016/j. str.2011.05.015.

39. Shewmaker F, McGlinchey RP, Thurber KR, McPhie P, Dyda F, Tycko R, et al. The functional curli amyloid is not based on in-register parallel beta-sheet structure. J Biol Chem 2009; 284:25065-76; PMID:19574225; http://dx.doi.org/10.1074/jbc.M109.007054.

40. Collinson SK, Parker JM, Hodges RS, Kay WW. Structural predictions of AgfA, the insoluble fimbrial subunit of Salmonella thin aggregative fimbriae. J Mol Biol 1999; 290:741-56; PMID:10395827; http:// dx.doi.org/10.1006/jmbi.1999.2882.

41. Wang X, Chapman MR. Sequence determinants of bacterial amyloid formation. J Mol Biol 2008; 380:570 80; PMID:18565345; http://dx.doi.org/10.1016/j. jmb.2008.05.019.

42. Wang X, Zhou Y, Ren JJ, Hammer ND, Chapman MR. Gatekeeper residues in the major curlin subunit modulate bacterial amyloid fiber biogenesis. Proc Natl Acad Sci USA 2010; 107:163-8; PMID:19966296; http://dx.doi.org/10.1073/pnas.0908714107.

43. Evans CG, Wisen S, Gestwicki JE. Heat shock proteins 70 and 90 inhibit early stages of amyloid beta-(1-42) aggregation in vitro. J Biol Chem 2006; 281:3318291; PMID:16973602; http://dx.doi.org/10.1074/jbc. M606192200.

44. Huang C, Cheng H, Hao S, Zhou H, Zhang X, Gao $\mathrm{J}$, et al. Heat shock protein 70 inhibits alpha-synuclein fibril formation via interactions with diverse intermediates. J Mol Biol 2006; 364:323-36; PMID:17010992; http://dx.doi.org/10.1016/j.jmb.2006.08.062.

45. Yoshiike Y, Minai R, Matsuo Y, Chen YR, Kimura T, Takashima A. Amyloid oligomer conformation in a group of natively folded proteins. PLoS ONE 2008; 3:3235; PMID:18800165; http://dx.doi.org/10.1371/ journal.pone. 0003235 .

46. Bardwell JC, Craig EA. Major heat shock gene of Drosophila and the Escherichia coli heat-inducible dnaK gene are homologous. Proc Natl Acad Sci USA 1984; 81:848-52; PMID:6322174; http://dx.doi. org/10.1073/pnas.81.3.848.

47. Bukau B, Horwich AL. The Hsp70 and Hsp60 chaperone machines. Cell 1998; 92:351-66; PMID:9476895 http://dx.doi.org/10.1016/S0092-8674(00)80928-9.

48. Zolkiewski M. ClpB cooperates with DnaK, DnaJ and GrpE in suppressing protein aggregation. A novel multi-chaperone system from Escherichia coli. J Biol Chem 1999; 274:28083-6; PMID:10497158; http:// dx.doi.org/10.1074/jbc.274.40.28083.

49. Flaherty KM, Wilbanks SM, DeLuca-Flaherty C, McKay DB. Structural basis of the 70-kilodalton heat shock cognate protein ATP hydrolytic activity. II. Structure of the active site with ADP or ATP bound to wild type and mutant ATPase fragment. J Biol Chem 1994; 269:12899-907; PMID:8175707.

50. Schmid D, Baici A, Gehring H, Christen P. Kinetics of molecular chaperone action. Science 1994; 263:9713; PMID:8310296; http://dx.doi.org/10.1126/science. 8310296 .
51. Szabo A, Langer T, Schroder H, Flanagan J, Bukau B, Hartl FU. The ATP hydrolysis-dependent reaction cycle of the Escherichia coli Hsp70 system DnaK, DnaJ and GrpE. Proc Natl Acad Sci USA 1994; 91:103459; PMID:7937953; http://dx.doi.org/10.1073/ pnas.91.22.10345.

52. Packschies L, Theyssen H, Buchberger A, Bukau B, Goody RS, Reinstein J. GrpE accelerates nucleotide exchange of the molecular chaperone DnaK with an associative displacement mechanism. Biochemistry 1997; 36:3417-22; PMID:9131990; http://dx.doi. org/10.1021/bi9628351.

53. Russell R, Wali Karzai A, Mehl AF, McMacken R. DnaJ dramatically stimulates ATP hydrolysis by DnaK: insight into targeting of $\mathrm{Hsp} 70$ proteins to polypeptide substrates. Biochemistry 1999; 38:416576; PMID:10194333; http://dx.doi.org/10.1021/ bi9824036.

54. Schröder H, Langer T, Hartl FU, Bukau B. DnaK, DnaJ and GrpE form a cellular chaperone machinery capable of repairing heat-induced protein damage. EMBO J 1993; 12:4137-44; PMID:7900997.

55. Jakob U, Muse W, Eser M, Bardwell JC. Chaperone activity with a redox switch. Cell 1999; 96:341-52; PMID:10025400; http://dx.doi.org/10.1016/S00928674(00)80547-4.

56. Chuang SE, Blattner FR. Characterization of twentysix new heat shock genes of Escherichia coli. J Bacterio 1993; 175:5242-52; PMID:8349564.

57. Janda I, Devedjiev Y, Derewenda U, Dauter Z, Bielnicki J, Cooper DR, et al. The crystal structure of the reduced, $\mathrm{Zn}^{2+}$-bound form of the B. subtilis $\mathrm{Hsp} 33$ chaperone and its implications for the activation mechanism. Structure 2004; 12:1901-7; PMID:15458638; http://dx.doi.org/10.1016/j.str.2004.08.003.

58. Won HS, Low LY, Guzman RD, Martinez-Yamout M, Jakob U, Dyson HJ. The zinc-dependent redox switch domain of the chaperone $\mathrm{Hsp} 33$ has a novel fold. J Mol Biol 2004; 341:893-9; PMID:15328602; http:// dx.doi.org/10.1016/j.jmb.2004.06.046.

59. Graumann J, Lilie H, Tang X, Tucker KA, Hoffmann $\mathrm{JH}$, Vijayalakshmi J, et al. Activation of the redox-regulated molecular chaperone Hsp33 - a two-step mechanism. Structure 2001; 9:377-87; PMID:11377198; http://dx.doi.org/10.1016/S0969-2126(01)00599-8.

60. Ilbert M, Horst J, Ahrens S, Winter J, Graf PC, Lilie $\mathrm{H}$, et al. The redox-switch domain of Hsp33 functions as dual stress sensor. Nat Struct Mol Biol 2007; 14:55663; PMID:17515905; http://dx.doi.org/10.1038/ nsmb1244.

61. Winter J, Ilbert M, Graf PC, Ozcelik D, Jakob $\mathrm{U}$. Bleach activates a redox-regulated chaperone by oxidative protein unfolding. Cell 2008; 135:691701; PMID:19013278; http://dx.doi.org/10.1016/j. cell.2008.09.024.

62. Quan S, Koldewey P, Tapley T, Kirsch N, Ruane KM, Pfizenmaier J, et al. Genetic selection designed to stabilize proteins uncovers a chaperone called Spy. Nat Struct Mol Biol 2011; 18:262-9; PMID:21317898; http://dx.doi.org/10.1038/nsmb.2016.

63. Bury-Moné S, Nomane Y, Reymond N, Barbet R, Jacquet E, Imbeaud S, et al. Global analysis of extracytoplasmic stress signaling in Escherichia coli. PLoS Genet 2009; 5:1000651; PMID:19763168; http:// dx.doi.org/10.1371/journal.pgen.1000651.

64. MacRitchie DM, Buelow DR, Price NL, Raivio TL. Two-component signaling and gram negative envelope stress response systems. Adv Exp Med Biol 2008; 631:80-110; PMID:18792683; http://dx.doi. org/10.1007/978-0-387-78885-2_6.

65. Price NL, Raivio TL. Characterization of the Cpx regulon in Escherichia coli strain MC4100. J Bacteriol 2009; 191:1798-815; PMID:19103922; http://dx.doi. org/10.1128/JB.00798-08

66. Raffa RG, Raivio TL. A third envelope stress signal transduction pathway in Escherichia coli. Mol Microbiol 2002; 45:1599-611; PMID:12354228; http://dx.doi.org/10.1046/j.1365-2958.2002.03112.x. 
67. DePace AH, Santoso A, Hillner P, Weissman JS. A critical role for amino-terminal glutamine/asparagine repeats in the formation and propagation of a yeast prion. Cell 1998; 93:1241-52; PMID:9657156; http:// dx.doi.org/10.1016/S0092-8674(00)81467-1.

68. Rüdiger S, Mayer MP, Schneider-Mergener J, Bukau B. Modulation of substrate specificity of the DnaK chaperone by alteration of a hydrophobic arch. J Mol Biol 2000; 304:245-51; PMID:1 1090270; http://dx.doi. org/10.1006/jmbi.2000.4193.

69. Collinson SK, Emody L, Muller KH, Trust TJ, Kay WW. Purification and characterization of thin, aggregative fimbriae from Salmonella enteritidis. J Bacteriol 1991; 173:4773-81; PMID:1677357.

70. LeVine $\mathrm{H}$. Thioflavine $\mathrm{T}$ interaction with synthetic Alzheimer disease beta-amyloid peptides: detection of amyloid aggregation in solution. Protein Sci 1993; 2:404-10; PMID:8453378.

71. LeVine H. Quantification of beta-sheet amyloid fibril structures with thioflavin T. Methods Enzymo 1999; 309:274-84; PMID:10507030; http://dx.doi. org/10.1016/S0076-6879(99)09020-5.

72. Kayed R, Head E, Thompson JL, McIntire TM, Milton SC, Cotman CW, et al. Common structure of soluble amyloid oligomers implies common mechanism of pathogenesis. Science 2003; 300:4869; PMID:12702875; http://dx.doi.org/10.1126/science.1079469.

73. Shorter J, Lindquist S. Hsp 104 catalyzes formation and elimination of self-replicating Sup35 prion conformers. Science 2004; 304:1793-7; PMID:15155912; http:// dx.doi.org/10.1126/science. 1098007.

74. Lotz GP, Legleiter J, Aron R, Mitchell EJ, Huang $\mathrm{SY}, \mathrm{Ng} \mathrm{C}$, et al. Hsp70 and Hsp40 functionally interact with soluble mutant huntingtin oligomers in a classic ATP-dependent reaction cycle. J Biol Chem 2010; 285:38183-93; PMID:20864533; http://dx.doi. org/10.1074/jbc.M110.160218.
75. DiGiuseppe PA, Silhavy TJ. Signal detection and target gene induction by the CpxRA two-component system. J Bacteriol 2003; 185:2432-40; PMID:12670966 http://dx.doi.org/10.1128/JB.185.8.2432-40.2003.

76. Thede GL, Arthur DC, Edwards RA, Buelow DR Wong JL, Raivio TL, et al. Structure of the periplasmic stress response protein CpxP. J Bacterio 2011; 193:2149-57; PMID:21317318; http://dx.doi. org/10.1128/JB.01296-10.

77. Rizzitello AE, Harper JR, Silhavy TJ. Genetic evidence for parallel pathways of chaperone activity in the periplasm of Escherichia coli. J Bacteriol 2001; 183:6794800; PMID:11698367; http://dx.doi.org/10.1128/ JB.183.23.6794-800.2001.

78. Sklar JG, Wu T, Kahne D, Silhavy TJ. Defining the roles of the periplasmic chaperones SurA, Skp and DegP in Escherichia coli. Genes Dev 2007; 21:2473 84; PMID:17908933; http://dx.doi.org/10.1101/ gad. 1581007

79. Kumita JR, Poon S, Caddy GL, Hagan CL, Dumoulin $\mathrm{M}$, Yerbury JJ, et al. The extracellular chaperone clusterin potently inhibits human lysozyme amyloid formation by interacting with prefibrillar species. J Mol Bio 2007; 369:157-67; PMID:17407782; http://dx.doi org/10.1016/j.jmb.2007.02.095.

80. Raman B, Ban T, Sakai M, Pasta SY, Ramakrishna T, Naiki $\mathrm{H}$, et al. AlphaB-crystallin, a small heat-shock protein, prevents the amyloid fibril growth of an amyloid beta-peptide and beta2-microglobulin. Biochem J 2005; 392:573-81; PMID:16053447; http://dx.doi. org/10.1042/BJ20050339.
81. Yerbury JJ, Poon S, Meehan S, Thompson B, Kumita JR, Dobson CM, et al. The extracellular chaperone clusterin influences amyloid formation and toxicity by interacting with prefibrillar structures. FASEB J 2007; 21:2312-22; PMID:17412999; http://dx.doi. org/10.1096/fj.06-7986com.

82. Hardy J, Selkoe DJ. The amyloid hypothesis of Alzheimer disease: progress and problems on the road to therapeutics. Science 2002; 297:353-6; PMID:12130773; http://dx.doi.org/10.1126/science. 1072994 .

83. Dueholm MS, Nielsen SB, Hein KL, Nissen Chapman M, Christiansen G, et al. Fibrillation of the Major Curli Subunit CsgA under a Wide Range of Conditions Implies a Robust Design of Aggregation. Biochemistry 2011; 50:8281-90; PMID:21877724, http://dx.doi.org/10.1021/bi200967c.

84. Ruschak AM, Miranker AD. Fiber-dependent amyloid formation as catalysis of an existing reaction pathway. Proc Natl Acad Sci USA 2007; 104:12341 6; PMID:17640888; http://dx.doi.org/10.1073/ pnas.0703306104.

85. Barnhart MM, Chapman MR. Curli biogenesis and function. Annu Rev Microbiol 2006; 60:131-47; PMID:16704339; http://dx.doi.org/10.1146/annurev. micro.60.080805.142106

86. Skowyra D, Wickner S. The interplay of the GrpE heat shock protein and $\mathrm{Mg}^{2+}$ in RepA monomerization by DnaJ and DnaK. J Biol Chem 1993; 268:25296-301; PMID:8244960.
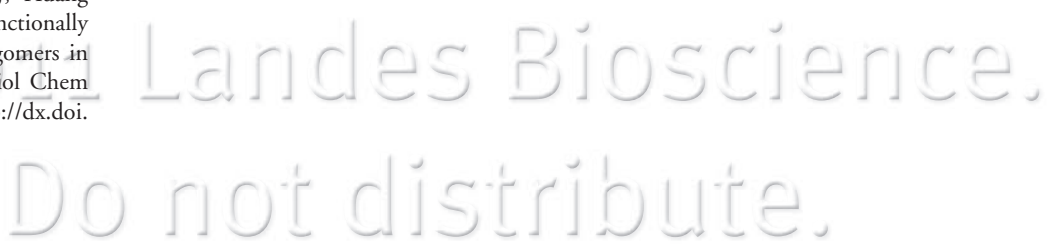